\title{
Phase separation in effective hard-core boson and triplet models in one and two dimensions
}

\author{
José A. Riera \\ Instituto de Fúsica Rosario, Consejo Nacional de Investigaciones Científicas y Técnicas, y Departamento de Fúsica, \\ Universidad Nacional de Rosario, Avenida Pellegrini 250, 2000-Rosario, Argentina
}

(November 19, 2018)

\begin{abstract}
Effective models of hard-core hole pair bosons and triplets are derived from the $t$ - $J$ model in ladders and on the square lattice by performing a change from site to dimer basis. The Hilbert space is truncated by projecting out single-occupied electron states and only nearest neighbor interactions are retained. The resulting effective models in one and two dimensions are studied by numerical techniques. In both spatial dimensions, the main result is that each hole pair is surrounded by a singlet cloud expelling triplet excitations from its vicinity. It is suggested an interpretation of this feature as a phase separated state between a hole-pair rich singlet phase and an undoped triplet phase with antiferromagnetic correlations. The possible relevance of this result to other theoretical scenarios and experimental results is discussed.
\end{abstract}

PACS: 74.20.-z, 74.25.Dw, 74.25.Ha, 74.20.Mn

\section{INTRODUCTION}

The $\mathrm{SO}(5)$ theoryt is a very appealing framework in which antiferromagnetic (AF) and superconducting (SC) phases of high- $\mathrm{T}_{c}$ superconductors are naturally related. This theory not only gives an explanation of the resonant peak observed in the SC phase but also predicts that it smoothly connects with the magnon peak of the $\mathrm{AF}$ phase as it was recently observed experimentally. 3 Another of the predictions of this theory, i.e. the presence of $\mathrm{AF}$ order inside the vortices present when a magnetic field is applied on the SC phase has recently motivated a flurry of experimental activity prediction. At the realm of the $\mathrm{SO}(5)$ theory lies the fact of singlet pairing of electrons. This is a deeply strongly correlated electrons feature yhich shares with resonant valence bond (RVB) theories 9 and excludes an explanation of the most important features of SC cuprates, not only the $\mathrm{AF}$ and $\mathrm{SC}$ phases but also the intervening pseudogap phase, via Fermi liquid or Fermi liquid instability concepts. Although there have been many attempts of formulating a $\mathrm{SO}(5)$ symmetric model on lattices, 6.8 a connection between the continuum theory and a more microscopic model like the $t-J$ model is still missing.

In this sense, one of the motivations of the present study is to help bridge the gap between the more phenomenological $\mathrm{SO}(5)$ theory and the microscopic $t-J$ model. More specifically, our goal is to obtain and study an effective model obtained by a change of basis from the site, spin- $1 / 2$ electrons, basis to the dimer basis, and then projecting out single occupied dimer states. This dimer basis also indicates a relation with RVB scenarios, in particular with its nearest neighbor version. in the present work the change of basis is performed on a single spatial dimer covering and hence the problem of an overcomplete basis which affects RVB models is avoided. In addition, the elimination of single-occupied dimers implies that the excitations of our model will not be spinons and holons, resulting from the fractionalization of electrons, but, as in the $\mathrm{SO}(5)$ theory, collective states of singlet binding of electrons in dimers 1 As a matter of fact, a recent study of the spin- $1 / 2$ Heisenberg model in two dimensions (2D), starting from a singlet RVB "soup", has precisely arrived at the conclusion that the elementary excitations of the AF ground state are spin-1 excited dimers instead of spinons 10

The pseudogap phase11, to many a central piece of the puzzle of understanding high- $\mathrm{T}_{c}$ superconductivity, is a candidate to be a realization of a $\mathrm{SO}(5)$ symmetric state or an RVB state. An effective model on dimers could then be a good starting point to understand many features of the pseudogap phase. In addition, the stripe phase 12 , which in some cuprates appears inside the pseudogap phase, could be explained in terms of a particular ordering of dimers that is pinned by a spatiplly anisotropic exchange or by coupling to the lattice.13.14 In fact, the concept of electron singlets play a central role in one of the theories of the stripe phase 15

There is a more specific, recent experimental result which could be possibly described in terms of an effective model on dimers. A scanning tunneling microscopy study of $\mathrm{Bi}_{2} \mathrm{Sr}_{2} \mathrm{CaCu}_{2} \mathrm{O}_{8+\delta}$ has shown a phase separation between superconducting islands inside a percolating background with physical properties resembling those of the pseudogap phase. 16

Other motivations to study effective models are both technical and conceptual. From the methodological point of view the reduction of the Hilbert space is an advantage to most numerical techniques, exact diagonalization, density-matrix renormalization-group, and quantum Monte Carlo. Conceptually, the effective model may, as it is shown below, make evident some properties which are somewhat hidden in the original $t-J$ Hamiltonian.

As said above, our approach to $\mathrm{SO}(5)$ concepts is to map the 2D $t-J$ model onto an effective model in a basis of singlet electron dimers. Since the original model excludes 
double-occupied sites, this connection is actually closer to a recently proposed variant of the $\mathrm{SO}(5)$ medel called the "projected" $\mathrm{SO}(5)$ (or $\mathrm{pSO}(5)$ ) model.17, 18 This $\mathrm{pSO}(5)$ model is an attempt to close the bridge with a microscopic model on a lattice from the other end with respect to what is intended in the present work. Although the basis set of the $\mathrm{pSO}(5)$ model is identical to that of the models that will be shown below, there are important differences in the corresponding Hamiltonians.

The paper is organized as follows. In Section II, an effective model is derived for the $t-J$ model on ladders. In this case, the procedure can be carried on in a much cleaner way than for the $t-J$ model on the square lattice. In addition, the one-dimensional (1D) character of this effective model allows its study by exact diagonalization techniques. The main results are common to the corresponding ones for the most interesting case, i.e. on the square lattice, which are obtained in Section [II]. Finally, in the Conclusions, the possible relevance of the present results to understand a number of previous theoretical problems as well as experimental results is discussed.

\section{LADDERS}

Although the main interest in connection with high$\mathrm{T}_{c}$ superconductivity is to study the interplay between various strongly correlated electron phases in $2 \mathrm{D}$, it is instructive to start with the analysis of two-leg ladders. 19 It should be noticed that the $t-J$ model on this lattice has been initially studied, ampng various reasons, as a realization of an RVB state.20 In addition, extensive studies have revealed strong similarities between the behaviors obtained on ladders and on the $2 \mathrm{D}$ square lattice.

The Hamiltonian of the $t-J$ model is:

$\mathcal{H}=-\sum_{\langle i j\rangle, \sigma} t_{i j}\left(\tilde{c}_{i \sigma}^{\dagger} \tilde{c}_{j \sigma}+\right.$ h.c. $)+\sum_{\langle i j\rangle} J_{i j}\left(\mathbf{S}_{i} \cdot \mathbf{S}_{j}-\frac{1}{4} n_{i} n_{j}\right)$

where the notation is standard. On ladders, $t_{i j}=t, J_{i j}=$ $J$ along the legs, and $t_{i j}=t_{\perp}, J_{i j}=J_{\perp}$ on the rungs. On chains and on the square lattice, the isotropic and homogeneous case $\left(t_{i j}=t, J_{i j}=J\right)$ will be considered. (a)

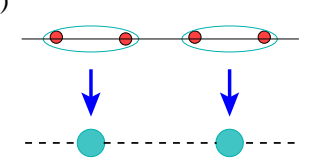

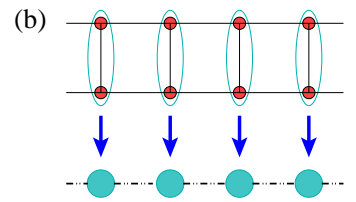

FIG. 1. Change from a site to a dimer basis in (a) a chain and (b) a ladder.

At half-filling, the exact change of basis from the site to the dimer basis (Fig. 1 1 (b)) leads to the following Hamiltonian:

$$
\mathcal{H}_{J, l a d d e r}^{(\text {dimer })}=2 J \sum_{\langle i, j\rangle}\left(t_{0, i}^{\dagger} t_{0, j}^{\dagger}-t_{+, i}^{\dagger} t_{-, j}^{\dagger}-t_{-, i}^{\dagger} t_{+, j}^{\dagger}+\text { h.c. }\right)
$$

$$
\begin{aligned}
& +2 J \sum_{\langle i, j\rangle, \alpha}\left(t_{\alpha, i}^{\dagger} t_{\alpha, j}+\text { h.c. }\right)+2 J \sum_{\langle i, j\rangle} \mathbf{S}_{i} \cdot \mathbf{S}_{j} \\
& +J_{\perp} \sum_{i}\left(n_{t, i}-1\right)
\end{aligned}
$$

where $t_{\alpha, i}^{\dagger}$ is a creation operator of a triplet with $S^{z}=$ $0,1,-1(\alpha=0,+,-)$ at dimer $i, n_{t, i}=n_{0, i}+n_{+, i}+n_{-, i}$, $n_{\alpha, i}=t_{\alpha, i}^{\dagger} t_{\alpha, i}$. The first term corresponds to the spontaneous creation of two triplets out of the vacuum with the constraint of keeping total $S^{z}=0$ and the second term is a triplet "hopping". It has been noted that this constraint is not satisfied in the $\mathrm{pSO}(5)$ Hamiltonian.22 The third term is the usual spin-1 Heisenberg exchange Hamiltonian. This Hamiltonian (or equivalent expressions) has been derived previously (e.g. Ref. 8, and references thereip) and used in restricted Hilbert space diagonalizations.21 The Hilbert space of the effective model turns out to be disconnected into two pieces: the set with even and the set of odd number of triplets. For example, in the subspace of total $S^{z}=0$ the first set is generated by successive applications of the Hamiltonian to the initial state (...sssss...), and the second one to the initial state (...sst $s s \ldots)$, where $s$ stands for a singlet dimer. This splitting of the Hilbert space does not appear in the effective model obtained for chains (Fig. 1 $1(\mathrm{a})$ ).

Away from half-filling, the states with a single hole occupancy on a dimer are projected out. If $\mathcal{P}$ is the projection operator on the subspace of retained states and $\mathcal{Q}$ is the projection operator on the subspace of the eliminated states, then the effective Hamiltonian is given by the standard formula:

$$
\mathcal{H}_{\text {eff }}=\mathcal{P H \mathcal { P }}-\mathcal{P} \mathcal{H} \mathcal{Q} \frac{1}{\mathcal{Q H \mathcal { Q }}-E_{0}} \mathcal{Q H \mathcal { P }}
$$

(1) where $\mathcal{H} \Psi_{0}=E_{0} \Psi_{0}$, and $\mathcal{H}_{\text {eff }} \mathcal{P} \Psi_{0}=E_{0} \mathcal{P} \Psi_{0}$. If $\mathcal{H}$ is the one given by Eq. (1) on the $2 \times 2$ ladder with 2 holes, the effective Hamiltonian will contain nearest neighbor $(\mathrm{NN})$ terms only. In this way, the hopping term of the effective Hamiltonian, $\mathcal{H}_{t, \text { eff }}$ results:

$$
\begin{aligned}
\mathcal{H}_{t, l a d d e r}^{(e f f)}= & -t_{s} \sum_{\langle i, j\rangle}\left(b_{j}^{\dagger} b_{i}+\text { h.c. }+n_{p, j} n_{s, i}+n_{s, j} n_{p, i}\right) \\
& -t_{t} \sum_{\langle i, j\rangle, s}\left(b_{j}^{\dagger} t_{j, s} t_{i, s}^{\dagger} b_{i}+\text { h.c. }+n_{p, j} n_{t, i}+n_{t, j} n_{p, i}\right) \\
& +J_{\perp} \sum_{i}\left(n_{p, i}-1\right)
\end{aligned}
$$

where $b_{i}^{\dagger}$ is a creation operator of a hole pair in the dimer $i, n_{p, i}=b_{i}^{\dagger} b_{i} . \quad n_{s, i}=1,0$ if the site is empty (i.e., a singlet) or occupied (by a triplet or a pair) respectively. The first term corresponds to the hopping of a pair to a singlet site, while the second term corresponds to the hopping to a site occupied by a triplet. The coupling constants $t_{s}, t_{t}$ are complicated functions of the original 
parameters $\left\{J, J_{\perp}, t, t_{\perp}\right\}$ but always $t_{s}>t_{t}$, as shown in Fig. 2 for two values of the lattice anisotropy $a=t_{\perp} / t$ $\left(t_{s}=2 t_{t}\right.$, for the isotropic ladder $\left.a=1\right)$. This is true even in the presence of a NN pair-pair Coulomb repulsion. This relation between the hopping parameters already suggests one of the most important results of this work. That is, the pairs would tend to be surrounded by singlets rather than by triplets in order to gain kinetic energy. In addition, the Heisenberg term in Eq. (2) would favor the clustering of triplets. These two combined effects would imply the phase separation between a pair-doped singlet phase and an undoped antiferromagnetics phase.

Finally, the full effective Hamiltonian in the projected dimer basis is given by:

$$
\mathcal{H}_{\text {ladder }}^{(\text {eff })}=\mathcal{H}_{J, \text { ladder }}^{(\text {dimer })}+\mathcal{H}_{t, \text { ladder }}^{(\text {eff })}
$$

The 1D effective model can be studied by exact diagonalization techniques (Lanczos algorithm). Most of the results below were obtained on a $L=12$ chain with periodic boundary conditions. Some computations for a $L=16$ chain show that finite size effects are not important. All results shown below correspond to fixed number of pairs $N_{p}$, and were obtained for the ground state, $\mathbf{k}=(0,0)$. In the following, all energies and coupling constants are expressed in units of $t$ of the original $t$ - $J$ Hamiltonian Eq. (11).

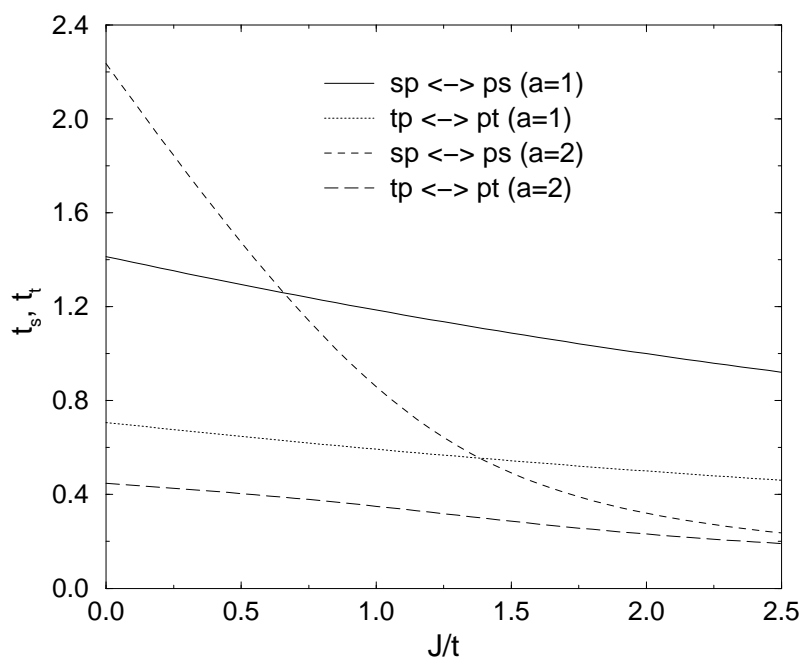

FIG. 2. Hopping amplitudes between pairs $p$ and singlets $s\left(t_{s}\right)$ and between hole pairs and triplets $t\left(t_{t}\right)$, as defined in Eq. (4), as a function of $J$ and for $a=1$ and 2. Basis change on a ladder (Fig. 11(b)).

In Fig. 3, the correlation between a pair and a triplet, $\left\langle n_{t} n_{p}\right\rangle$ at distance $r=1$ (NN sites) is shown for the $L=12$ chain with one pair, as a function of $J / t$ and for three ladder anisotropy ratios. It can be seen that the probability of finding a triplet next to a pair is much smaller than the probability of finding a pair next to a singlet. The later, in the one pair system, is simply $\left\langle n_{s} n_{p}\right\rangle=1-\left\langle n_{t} n_{p}\right\rangle$. It is more important the result that, as it can be easily seen in Fig. 3, $\left\langle n_{t} n_{p}\right\rangle(r=1)$ is always smaller than the triplet density $\left\langle n_{t}\right\rangle$ (inset) for the same $J / t$. The normalization of the later is such that $n_{s}+n_{t}+n_{p}=1, n_{p}=0.0833$ (one pair), and $n_{p}=0.1667$ (two pairs). This result suggests that triplets are expelled from the vicinity of a pair.

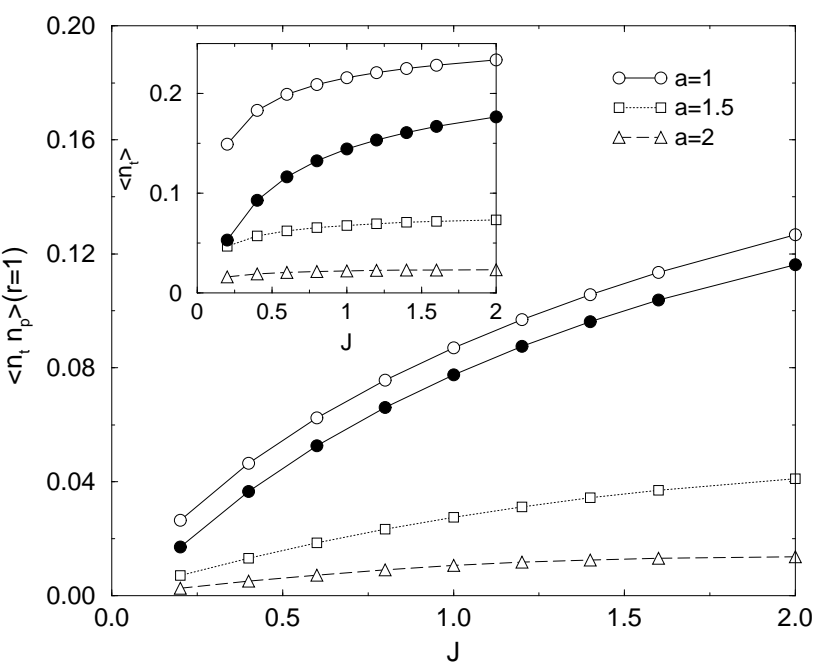

FIG. 3. Triplet-pair correlation at $r=1, L=12, N_{p}=1$ (open symbols), as a function of $J$ and for the values of the anisotropy ratio $a$ indicated on the plot. The inset shows the triplet density for the same cluster and parameters. Results for $N_{p}=2$ (filled circles), $a=1$ are also included.

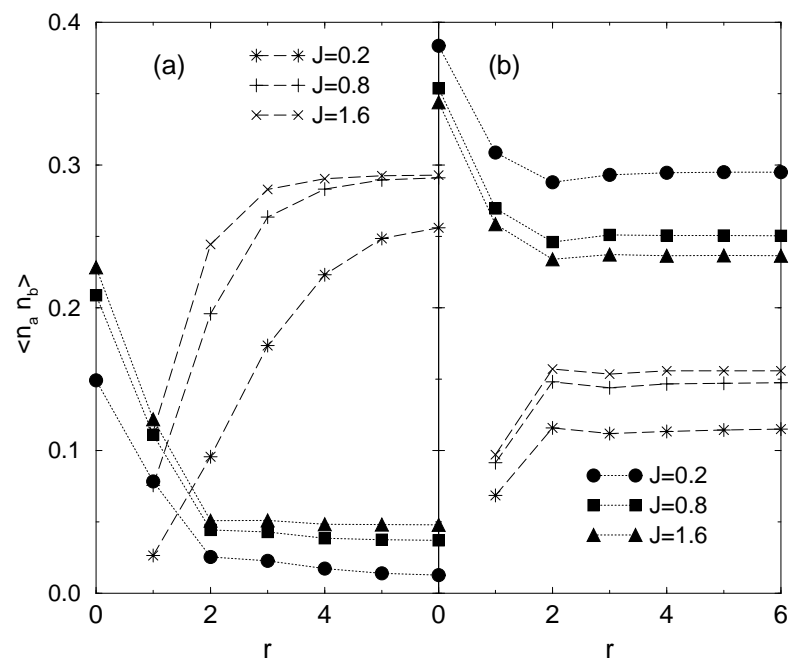

FIG. 4. Diagonal correlations as a function of distance obtained for $L=12, N_{p}=1, a=1$, and various values of $J$, as indicated on the plot. (a) Triplet-triplet (dot lines) and triplet-pair (dashed lines) correlations; (b) singlet-singlet (full symbols, divided by 2) and singlet-triplet (dashed lines) correlations. 
The complete picture can be inferred from Fig. \& by looking at various correlations $\left\langle n_{a} n_{b}\right\rangle, \quad(a, b=$ $\left.t_{0}, t_{+}, t_{-}, p\right)$ as a function of distance. From now on, the study will be limited to the isotropic case $\left(t=t_{\perp}, J=J_{\perp}\right.$ in the original $t-J$ model) but similar results were also found for all $a>1$ investigated. In this Figure, which corresponds also to the system with one pair, it can be observed that the triplet-triplet correlation is maximum at $r=0$, while the triplet-pair correlation is maximum at the largest distance on the chain. That is, triplets try to stay as far apart as possible form a pair. On the other hand, although singlet-singlet correlations are also maximum at $r=0$, the singlet-pair correlations are maximum at $r=0$. The final piece is that the singlet-triplet correlation is maximum at the maximum distance. Similar results were obtained for the larger $L=16$ site chain.

The picture emerging from these correlations is that pairs move preferentially in a background of singlets, and that both pairs and singlets try to keep themselves away from triplets. Thus, the system is separated between a pair-rich singlet phase (it is tempting to consider this phase as a doped "RVB" phase, even though that the present effective models are obtained for a single dimer covering) and a pair-poor triplet-rich phase which can be identified as an undoped AF phase.

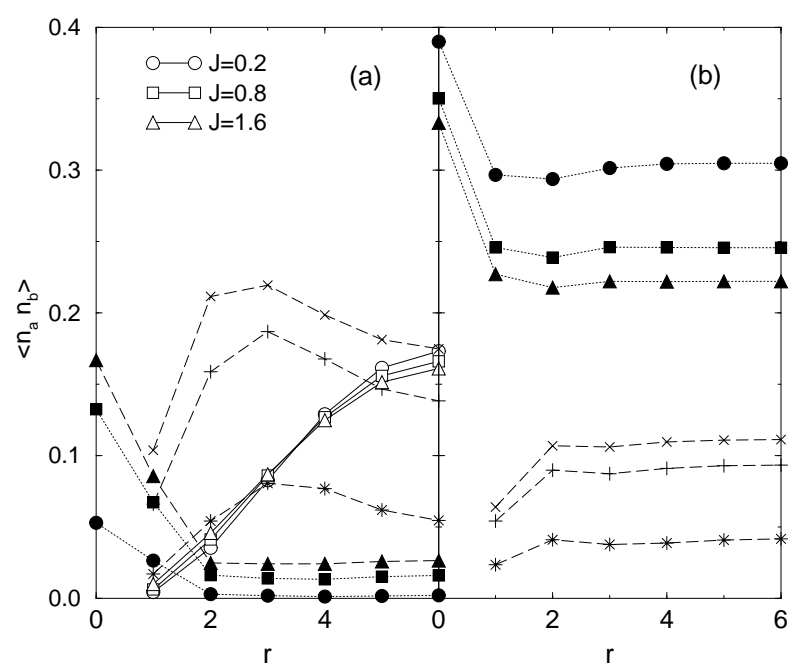

FIG. 5. Diagonal correlations as a function of distance obtained for $L=12, N_{p}=2, a=1$, and various values of $J$ as indicated on the plot. (a) Triplet-triplet (full symbols), pair-pair (open symbols) and triplet-pair (dashed lines) correlations; (b) singlet-singlet (full symbols, divided by 2) and singlet-triplet (dashed lines) correlations.

Essentially the same behavior is observed in the case of two pairs present in the system. Results for the tripletpair correlation in NN sites and the triplet density as a function of $J / t$ are also included in Fig. 3 for comparison with the one pair case. Both the probability of finding a pair next to a triplet and the triplet density are smaller than for the one pair case. A likely explanation of this behavior is that the introduction of more pairs in the system increases the volume of the pair-doped "RVB" phase leaving less room for the AF phase.

In Fig. 5, diagonal correlations are shown for $L=12$ and same parameters as in Fig. A. The new feature in Fig. 5 with respect to Fig. 4 is the fact that two pairs repel themselves as can be read from the fact that pair-pair correlations are maximum at the largest distance. Then, the same qualitative behavior found in the one-pair case holds: the system separates into a pair-doped singlet region, here formed by two islands and a pair-poor tripletrich region, presumably with short-range AF order, in this case filling the space between those two islands. Notice also in Fig. 5(b) the singlet-singlet correlations falling down to its bulk value within a lattice spacing.

\section{TWO DIMENSIONS}

The most important situation is that of the square lattice, which corresponds to the $\mathrm{CuO}_{2}$ planes in superconducting cuprates. There are again infinitely many different ways in which a change from the site to the dimer basis can be performed. One of them is shown in Fig. 6. A well-known feature of choosing a dimer basis like the one depicted in Fig. 6 is that the rotation invariance of the square lattice is broken and it is not simple to restore it at the level of the effective Hamiltonian. In the ladder case, examined in the previous Section, this is not important since the lattice itself is spatially anisotropic. In the case of the square lattice, the purpose of the present study is to provide indications of the presence, in a rotationally-broken effective model for the square lattice, of the singlet-AF phase separation already observed in ladders and to suggest that this feature should be also present in a rotational invariant formulation.23

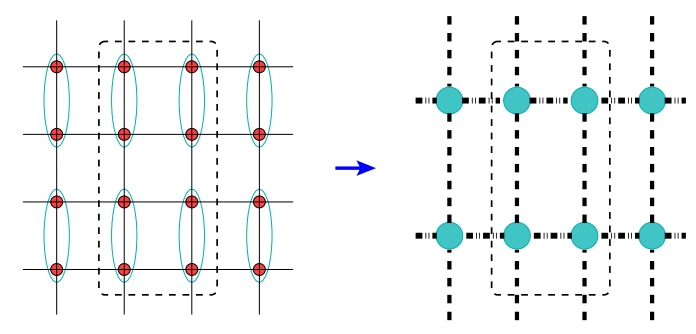

FIG. 6. A possible change from a site to a dimer basis in the square lattice. Dotted and dash-dotted lines indicate effective magnetic interactions in the new basis.

At half-filling the change of basis implies an exact mapping of the original spin- $1 / 2$ Heisenberg model onto a model written in terms of triplets and singlets. In the basis change indicated in Fig. 6 the resulting model is anisotropic with ladder-like interactions (Fig. 1 (b)) given by Eq. (2) in the horizontal direction and chain-like interactions (Fig. 11(a)) in the vertical direction, which are 
given by the following Hamiltonian:

$$
\begin{aligned}
\mathcal{H}_{J, \text { chain }}^{(\text {dime })}= & -J \sum_{\langle i, j\rangle}\left(t_{0, i}^{\dagger} t_{0, j}^{\dagger}-t_{+, i}^{\dagger} t_{-, j}^{\dagger}-t_{-, i}^{\dagger} t_{+, j}^{\dagger}+\text { h.c. }\right) \\
& +J \sum_{\langle i, j, \alpha\rangle}\left(t_{\beta, i}^{\dagger} t_{\gamma, j}^{\dagger} t_{\alpha, i}-t_{\gamma, i}^{\dagger} t_{\beta, j}^{\dagger} t_{\alpha, i}+\text { h.c. }\right) \\
& -J \sum_{\langle i, j\rangle, \alpha}\left(t_{\alpha, i}^{\dagger} t_{\alpha, j}+\text { h.c. }\right)+J \sum_{\langle i, j\rangle} \mathbf{S}_{i} \cdot \mathbf{S}_{j} \\
& +J_{\perp} \sum_{i}\left(n_{t, i}-1\right)
\end{aligned}
$$

where $\beta=+, 0,-, \gamma=-,+, 0$ for $\alpha=0,+,-$ respectively. The sign of the second term depends on the definition of the singlet. It should be noticed that this term, as in the ladder case, locally conserves the total $S^{z}$. This Hamiltonian can be read, with a slightly different notation though, in Ref. 10, and alternative or similar derivations can be found in several other places. 4321 Finally, the exchange part of the Hamiltonian in the dimer basis is:

$$
\mathcal{H}_{J, \text { square }}^{(\text {dimer })}=\mathcal{H}_{J, \text { ladder }}^{(\text {dimer })}+\mathcal{H}_{J, \text { chain }}^{(\text {dimer })}
$$

with the ladder (chain) term acting on the horizontal (vertical) direction as indicated in Fig. 6 .

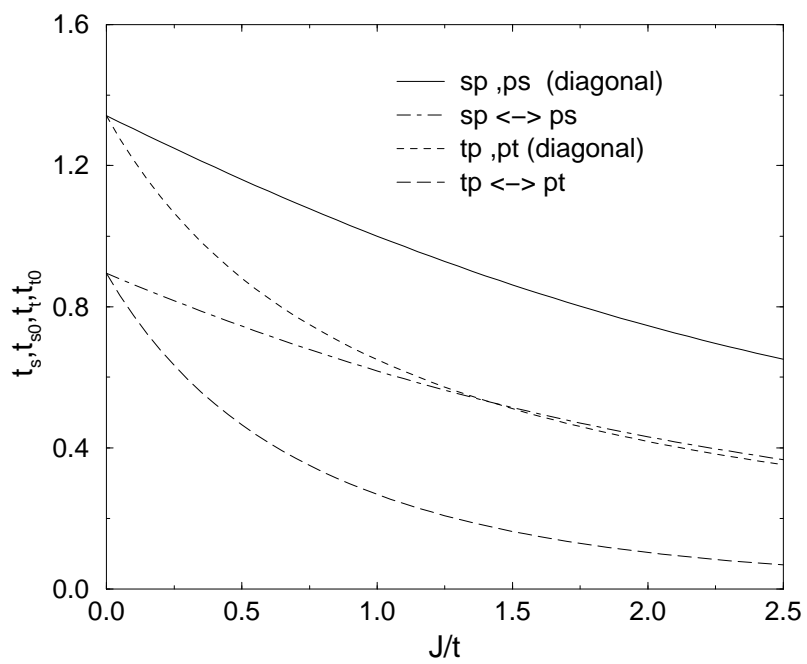

FIG. 7. Hopping amplitudes between pairs $p$ and singlets $s$ $\left(t_{s}, t_{s 0}\right)$ and between pairs and triplets $t\left(t_{t}, t_{t 0}\right)$, as defined in Eq. (8), as a function of $J$. Basis change on a chain (Fig. 1(a)).

Let us consider the original $t$ - $J$ Hamiltonian defined on a chain. To obtain the hopping part of the effective model, the projection defined by Eq. (3) is again used. In this case, $\mathcal{H}$ is the $t-J$ Hamiltonian on a 4 -site chain (Fig. 1(a)) with two holes and the effective one will contain $\mathrm{NN}$ interactions only. The resulting effective hopping term is very similar to the one in Eq. (何):

$$
\begin{array}{r}
\mathcal{H}_{t, c h a i n}^{(e f f)}=-t_{s}^{\prime} \sum_{\langle i, j\rangle}\left(b_{j}^{\dagger} b_{i}+\text { h.c. }\right) \\
-t_{s 0} \sum_{\langle i, j\rangle}\left(n_{p, j} n_{s, i}+n_{s, j} n_{p, i}\right) \\
-t_{t}^{\prime} \sum_{\langle i, j\rangle, s}\left(b_{j}^{\dagger} t_{j, s} t_{i, s}^{\dagger} b_{i}+\text { h.c. }\right) \\
-t_{t 0} \sum_{\langle i, j\rangle}\left(n_{p, j} n_{t, i}+n_{t, j} n_{p, i}\right) \\
+J_{\perp} \sum_{i}\left(n_{p, i}-1\right)
\end{array}
$$

As in the case of Eq. (14), the hopping amplitudes satisfy $t_{s}^{\prime}>t_{t}^{\prime}$, and $t_{s 0}>t_{t 0}$ (Fig. 17) again favoring the movement of pairs away from triplet-rich regions.

Coming back to the square lattice, the correct procedure is to to take $\mathcal{H}$ as the $t-J$ Hamiltonian on the eight-site cluster indicated by a dashed box in Fig. 6. As a result, the effective Hamiltonian contains three- and four-site terms in addition to NN interactions. In order to keep the Hamiltonian close as possible with the proposed $\mathrm{pSO}(5)$ model 17.18 only NN hopping interactions as given by Eqs. (14) and (\$) in the horizontal and vertical directions respectively are retained. The signs of these hopping terms coming from the eight-site cluster calculation are the same as in Eqs. (4) and (8). To compensate for neglecting three- and four-site terms, the hopping amplitudes in (4) are re-scaled by a single constant $\alpha$ and the amplitudes in (8) by another constant $\beta$. A reasonable fit of the energies of the effective model on the $4 \times 2$ cluster to the exact energies of the $t$ - $J$ model on the $4 \times 4$ cluster in the whole range studied, $0 \leq J \leq 2.5$, is achieved with $\alpha=1$ and $\beta=0.5$. In this range of $J$, the relative difference between these two energies is less than 0.01. The exchange part of the effective model is given by Eq.( (7).

A first insight on the properties of the effective model can be gained by studying the $4 \times 4$ cluster. Due to the large dimension of the Hilbert space $\left(\approx 7.7610^{7}\right.$ for $N_{p}=1$ and $\approx 1.5010^{8}$ for $N_{p}=2$ taking into account translational invariance), conventional exact diagonalization techniques cannot be applied, except by resorting to massive computers. Alternatively, a diagonalization in a systematically expanded Hilbert space (SEHS) 25 is used. With a number of states $\approx 510^{6}$, variational energies within $1 \%$ of the exact energies, estimated by extrapolating to the full dimension of the Hilbert space, are obtained. In order to study larger clusters, a quantum Monte Carlo(QMC) technique with the conventional worldline checkerboard decomposition 24 is used. Although there are no fermions involved, there is a "minus sign problem" which makes impossible the study at low temperatures. There are several terms in the effective Hamiltonian which lead to "minus sign" configurations in the 2+1-dimensional space. An important reduction of this problem is achieved by not generating those configurations with interacting cubes which do not 
conserve the parity of the number of triplets on its top and bottom plaquettes. Of course, the QMC algorithm is no longer exact but nevertheless it provides a reasonable approximation to its exact behavior. By eliminating certain transitions, it might be possible that certain regions of the phase space are disconnected. To cope with this problem, at each temperature, results were averaged over at least four independent runs starting from different initial states. Variations in the values of the energy from different runs were somewhat larger than the statistical error of each run but nevertheless smaller than $1 \%$ in all cases. In addition, since the the present study concerns zero temperature properties, the simulations are restricted to the subspace of zero total magnetization and only local moves are included in the algorithm. Overall simulation error bars are approximately twice the size of the symbols used.

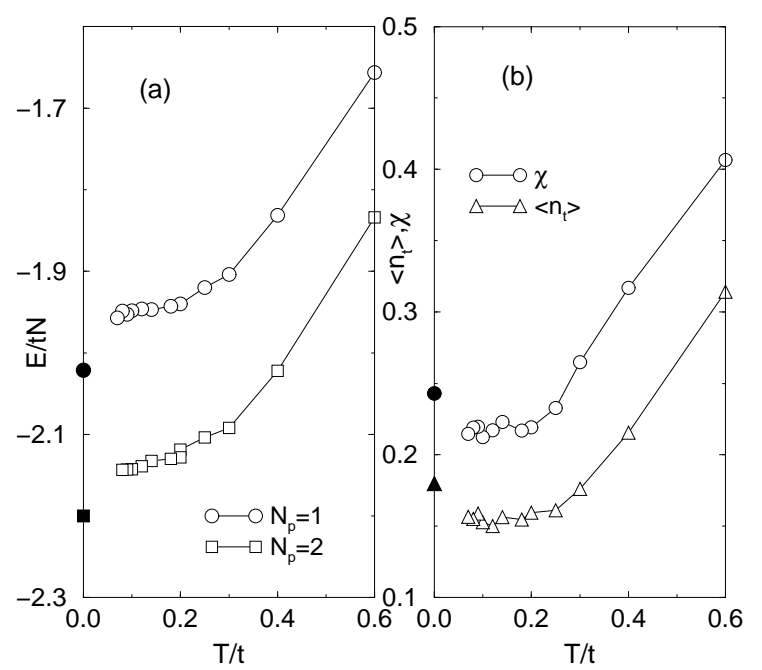

FIG. 8. (a) Variation of energy per site with temperature in the $S^{z}=0$ subspace in the $4 \times 4$ cluster with one and two pairs, $J / t=0.8$. (b) Variation of triplet density and $(\pi, \pi)$ magnetic structure factor with temperature in the subspace $S^{z}=0$ in the $4 \times 4$ cluster with two pairs, $J / t=0.8$. In both panels, solid symbols at $T=0$ indicate the corresponding values obtained by SEHS diagonalization.

Evolution of various quantities with temperature in the $S^{z}=0$ subspace on the $4 \times 4$ cluster, $J=0.8 t$, with one and two pairs are shown in Fig. 8. The corresponding zero temperature results obtained by diagonalization in an expanded Hilbert space are also included for comparison. As expected the energies obtained by the approximated QMC technique are higher than the obtained by diagonalization (which are virtually exact), while the triplet densities are smaller than the exact ones.

The same correlations previously studied on ladders are shown in Fig. 9(a) for the $4 \times 4$ cluster, with one and two pairs, $J=0.8 t$. These ground state correlations, obtained by SEHS at $\mathbf{k}=(0,0)$, have been averaged over the two-directions $x$ and $y$. The most important result is again that the triplets try to locate as far as possible from the pairs. Similar results were obtained for the other correlations shown in Fig. 月, and for all values of $J / t$ examined.

Results on the $8 \times 8$ cluster obtained by QMC technique at $\mathrm{T}=0.09 \mathrm{t}$ for one and two pairs are shown in Fig. 9(b). The behavior of triplet-triplet and triplet-pair correlations and pair-pair corrrelations are qualitatively similar to those found in the smaller cluster at zero temperature. These results are very suggestive that triplets are expelled from the vicinity of a pair. Notice also that, as for ladders (Fig. 5), singlet-singlet correlations decay very rapidly to their bulk value.

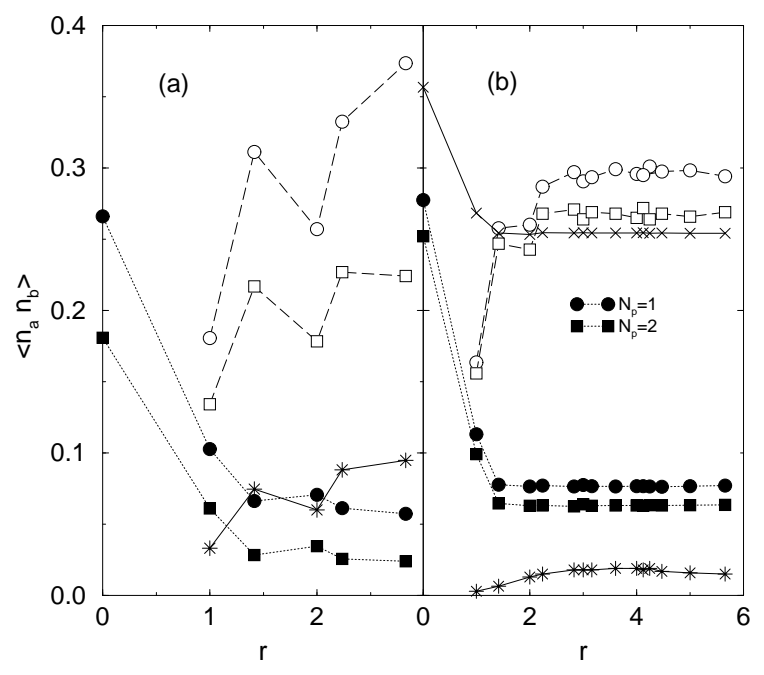

FIG. 9. Triplet-triplet (full symbols) and triplet-pair (open symbols) correlations as a function of distance, $N_{p}=1,2$, $J=0.8 t$, (a) on the $4 \times 4$ cluster (SEHS) and (b) on the $8 \times 8$ one (QMC, $\mathrm{T}=0.09 \mathrm{t}$ ). Pair-pair (stars) and singlet-singlet (crosses, (b) only) correlations for $N_{p}=2$ are also shown.

A more systematic study as a function of $J / t$ leads to the results depicted in Fig. 10(a). In all cases, the probability of finding a triplet near a pair (open symbols) is smaller than the average probability of finding a triplet on a given site (full symbols). Consistently with the idea of pairs expelling triplet excitations, the probability of finding a triplet at the largest distance of a pair (only shown for the one pair case) is larger than the average triplet probability. Singlet-singlet correlations vs. distance

Since the system contains charged pair bosons, it is important to calculate the superfluid density as a measure of superconducting properties in this model. To calculate the superfluid density it The procedure to calculate the superfluid density 26 starts by computing the correlation:

$$
C(\tau)=\left\langle\Delta p_{x}(\tau) \Delta p_{x}(0)\right\rangle
$$

where $\Delta p_{x}(\tau)=p_{x}(\tau+1)-p_{x}(\tau)$ and $p_{x}(\tau)=\sum x(i, \tau)$, where $x(i, \tau)$ is the $x$-coordinate of pair $i$ at imaginary 
time $\tau$ and the sum extends over all the pairs in the system. The superfluid density follows from:

$$
\rho_{s} \approx \lim _{\omega \rightarrow 0} \int_{0}^{\beta} d \tau C(\tau) \exp (-i \omega \tau)
$$

It is well-known that this quantity vanishes if the simulation is carried in a zero-winding number subspace which is the case in the present study. The way out of this problem stems from the fact that the winding number may be nonzero in half of the Trotter, imaginary time, direction, being also nonzero but with an opposite sign in the other half. The superfluid density then would come out by taking the Fourier transform in (10) between zero and $\beta / 2$. This procedure should be exact in the limit of infinite Trotter number which is the limit in which on the other hand the whole worldline algorithm is valid. This procedure has been thoroughly checked in the hardcore boson model where exact and numerical results are available.27,28

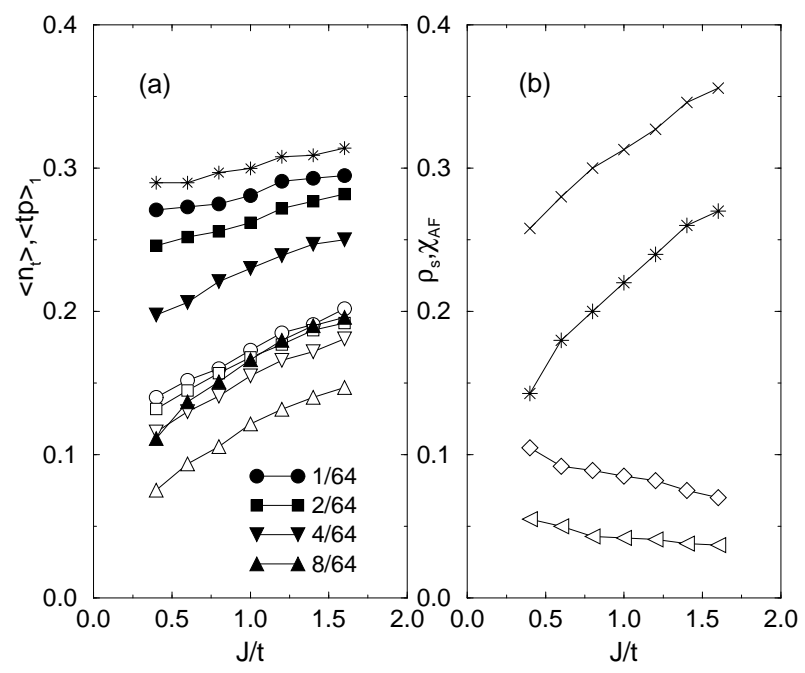

FIG. 10. Results obtained by QMC on the $8 \times 8$ cluster, $\mathrm{T}=0.09 \mathrm{t}$ at various fillings and as a function of $\mathrm{J} / \mathrm{t}$. (a) Triplet density (full symbols) and triplet-pair correlations at NN sites (open symbols). The triplet-pair correlation at the maximum distance for $N_{p}=1$ are shown with stars(b) AF structure factor (triangles: $N_{p}=4$, diamonds: $N_{p}=8$ ) and superfluid density (plus: $N_{p}=4$, stars: $N_{p}=8$ ).

The superfluid density, $\rho_{s}$, and the magnetic structure factor at $(\pi, \pi), \chi_{A F}$, on the $8 \times 8$ cluster with four and eight pairs, are shown in Fig. 10(b) as a function of $J / t$. Although the ultimate dominance of $\mathrm{SC}$ or $\mathrm{AF}$, or eventually the coexistence of both $\mathrm{SC}$ and $\mathrm{AF}$ orders can be determined only by a finite size extrapolation which is out of the scope of the present study, two qualitative features are apparent. First, as a pair doping is increased there is an enhancement of SC and a suppression of AF. Second, as $J / t$ is increased $\chi_{A F}$ also increases while $\rho$ decreases. Similar results were obtained with an even simplifief model which mimics the spin triplets by spin doublets 22 and can be obtained by projecting out the $S^{z}=0$ component of the triplets, $t_{0}$, again by using (3). The mutually exclusive behavior of AF and superconductivity, which has been shown both in the present work and previously in Ref. 22, can now be understood in terms of this real space phase separation between SCsinglet and AF regions. The growth of one of each phases reduces the space available for the other phase.

\section{CONCLUSIONS}

In the first place, the present work is concerned with the mapping of the $t-J$ model into an effective model of pairs and triplets moving on a "sea" of singlets. The strategy adopted was first to map exactly the magnetic interactions in the undoped system and then to determine the effective hopping interactions in the two-hole sector of the original Hamiltonian. This second procedure is an approximate one because it implies projecting out single-occupied dimers and truncating the range of the interactions nearest neighbors. In the case of ladders this procedure is reasonably clean. The choice of dimers corresponds to the strong coupling limit which extends virtually to the anisotropic case. An effective model in one dimension is obtained in which the hopping couplings between pairs and singlets are larger than between pairs and triplets.

In the square lattice the procedure to obtain the effective model is more complicated. In the first place, the simple dimer covering adopted breaks rotational invariance of the lattice. Still, the mapping of the magnetic interactions at half-filling is exact. In the second place, even by restricting the range of hoppings to nearest neighbors, three- and four-site interactions appear at the effective level. To eliminate these interactions another fit to the energies of a small $t$ - $J$ cluster was performed to rescale the effective hopping constants. The resulting effective hopping couplings show the same behavior as those obtained for the ladder case.

The resulting effective models were studied by numerical techniques, exact diagonalization in the case of the model obtained for the ladder lattice and approximate diagonalization and Quantum Monte Carlo for the square lattice case. Again the results obtained in the effective model for ladders are more clear than the ones obtained for the square lattice.

From the study of several correlations the following picture emerges. Pairs are surrounded predominantly by singlets, and triplet excitations are located as far apart as possible. This is a kind of phase separation between a pair-rich "RVB" phase and a pair-poor triplet-rich phase which would correspond to a phase with at least shortrange interactions (since triplet excitations restore $\mathrm{AF}$ order from a RVB state 19 ) and would quite likely be insulating. Pair-pair correlation functions indicate that 
pairs try to be situated at the maximum distance (this behavior is more definite in ladders than in 2D). This behavior might correspond to pairs moving in a singlet phase macroscopically separated from the triplet phase. Alternatively, the phase separation might be microscopic: pair-singlet islands moving in a triplet background. The behavior of triplet-pair correlations in 1D could favor this second scenario. In any case, these PS scenarios provide an explanation for the AF-SC mutual exclusion shown in the previous Section.

Further studies are necessary to distinguish between both types of phase separation. In the macroscopic PS scenario it is expected a superfluid density comparable with that of the hard-core boson model while on the microscopic PS scenario it would be considerable reduced. On theoretical grounds, the emerging PS picture could be realted to the AF-SC coexistence phase predicted by $\mathrm{SO}(5)$ theories. The exclusion of triplets and pairs is highly nontrivial taking into account that previous studies on the $t-J$ mode 29 have suggested a bound state between a $\mathrm{d}_{x^{2}-y^{2}}$ pair and a triplet. However, the internal structure of a pair, essential in the analysis of Ref. 29, is lost in the present study 30 Finally, it is also tempting to relate this PS state to recent observations of inhomogeneities in $\mathrm{Bi}_{2} \mathrm{Sr}_{2} \mathrm{CaCu}_{2} \mathrm{O}_{8+\delta}$ (Ref. 16. It might be possible that out-of-plane negative Coulomb centers could attract and pin pair-singlet islands in their surroundings.

\section{ACKNOWLEDGMENTS}

The author wishes to acknowledge many useful discussions with Prof. S. Maekawa and to Prof. M. Boninsegni for suggesting the procedure to compute the superfluid density.

${ }^{1}$ S.-C. Zhang, Science 275, 1089 (1997).

2 J. Rossat-Mignod, L. P. Regnault, C. Vettier, P. Bourges, P. Burlet, J. Bossy, J. Y. Henry, and G. Lapertot, Physica C 185-189, 86 (1991); H. A. Mook, M. Yethiraj, G. Aeppli, T. E. Mason, and T. Armstrong, Phys. Rev. Lett. 70, 3490 (1993); H. F. Fong, B. Keimer , D. Reznik , D. L. Milius, and I. A. Aksay, Phys. Rev. B 54, 6708 (1995).

${ }^{3} \mathrm{P}$. Bourges, in "The gap symmetry and Fluctuations in High Temperature Superconductors", ed. by J. Bok, G. Deutscher, D. Pavuna and S. A. Wolf (Plenum Press, 1998).

${ }^{4}$ B. Lake, G. Aeppli, K. N. Clausen, D. F. McMorrow, K. Lefman, N. E. Hussey, N. Mangkorntong, M. Nohara, H. Takegi, T. E. Mason, and A. Schröder, Science 291, 1759 (2001).

${ }^{5}$ J. E. Hoffman, E. W. Hudson, K. M. Lang, V. Madhavan, H. Eisaki, S. Uchida, and J. C. Davis, Science 295, 466 (2002).
${ }^{6}$ P. W. Anderson, Science 235, 1196 (1987).

${ }^{7}$ D. Scalapino, S.-C. Zhang, and W. Hanke, Phys. Rev. B 58, 443 (1998).

${ }^{8}$ R. Eder, A. Dorneich, M. G. Zacher, W. Hanke, and S. C. Zhang, Phys. Rev. B 59, 561 (1999).

${ }^{9}$ S. A. Kivelson, D. S. Rokhsar, and J. P. Sethna, Phys. Rev. B 35, 8865 (1987).

${ }^{10}$ R. Eder, Phys. Rev. B 59, 13810 (1999).

11 J. L. Tallon and J. W. Loram, Physica C 349, 53 (2001).

12 J. M.Tranquada, J. D. Axe, N. Ichikawa, Y. Nakamura, S. Uchida, and B. Nachumi, Phys. Rev. B 54, 7489 (1996); N. Ichikawa, S. Uchida, J. M. Tranquada, T. Niemoller, P. M. Gehring, S.-H. Lee, and J. R. Schneider, Phys. Rev. Lett. 85, 1738 (2000).

${ }^{13}$ K. Park and S. Sachdev, Phys. Rev. B 64, 184510 (2001).

${ }^{14}$ O. P. Sushkov, Phys. Rev. B 63, 174429 (2001).

${ }^{15}$ V. J. Emery, S. A. Kivelson, and O. Zachar, Phys. Rev. B 56,6120 (1996).

${ }^{16}$ K. M. Lang, V. Madhavan, J. E. Hoffman, E. W. Hudson, H. Eisaki, S. Uchida, and J. C. Davis, Nature 415, 412 (2002).

17 S. C. Zhang, J-P. Hu, E. Arrigoni, W. Hanke, and A. Auerbach, Phys. Rev. B 60, 13070 (1999).

18 A. Dorneich, W. Hanke, E. Arrigoni, M. Troyer, and S. C. Zhang, Phys. Rev. Lett. 88, 057003 (2002).

${ }^{19}$ E. Dagotto, J. Riera and D. Scalapino, Phys. Rev. B 45, 5744 (1992).

20 T. M. Rice, S. Gopalan and M. Sigrist, Europhys. Lett. 23, 445 (1993).

${ }^{21}$ E. Dagotto, G. B. Martins, J. Riera, A. L. Malvezzi, and C. Gazza, Phys. Rev. B 58, 12063 (1998).

22 J. A. Riera, Phys. Rev. B 65, 174526 (2002).

${ }^{23}$ E. Altman and A. Auerbach, Phys. Rev. B 65, 104508 (2002).

24 J. D. Reger and A. P. Young, Phys. Rev. B 37, 5978 (1988), and references therein.

25 José Riera, in High Performance Computing and its Applications in the Physical Sciences, edited by D. Browne, et al., (World Scientific, New York, 1994), pg. 72.

${ }^{26}$ G. G. Batrouni, R. T. Scalettar, and G. T. Zimanyi, Phys. Rev. Lett. 65, 1765 (1990).

${ }^{27}$ F. Hébert, G. G. Batrouni, R. T. Scalettar, G. Schmid, M. Troyer, and A. Dorneich, Phys. Rev. B 65, 014513 (2001).

${ }^{28}$ K. Bernardet, G. G. Batrouni, J.-L. Meunier, G. Schmid, M. Troyer, and A. Dorneich, Phys. Rev. B 65, 104519 (2002); G. Schmid, S. Todo, M. Troyer, and A. Dorneich, Phys. Rev. Lett. 88, 167208 (2002).

${ }^{29}$ J. Riera and E. Dagotto, Phys. Rev. B 57, 8609 (1998).

${ }^{30}$ An effective model retaining more details of the hole pairs can be seen in T. Siller, M. Troyer, T. M. Rice, and S. R. White, Phys. Rev. B 63, 195106 (2001). 